\title{
Effectiveness of sucrose analgesia in newborns undergoing painful medical procedures
}

\author{
Anna Taddio BScPhm PhD, Vibhuti Shah MD MSc, Rebecca Hancock MSc, \\ Ryan W. Smith BASc MSc, Derek Stephens MSc, Eshetu Atenafu MSc, Joseph Beyene PhD, \\ Gideon Koren MD, Bonnie Stevens RN PhD, Joel Katz PhD
}

$\infty$ See related research paper by Farion and colleagues, page 31, and related commentary by Anand, page 11

\section{ABSTRACT}

Background: Sucrose is widely used to manage procedural pain in term newborns despite a lack of evidence of its effectiveness for different procedures and infant populations. Our objectives were to evaluate the effectiveness and safety of sucrose in newborns undergoing various medical procedures within 2 days of birth.

Methods: We performed a double-blind, randomized controlled trial. We included newborns ( $\geq 36$ weeks gestation) of diabetic mothers and nondiabetic mothers. Each newborn received $2 \mathrm{~mL}$ of a $24 \%$-sucrose or placebo solution before all procedures. We used the Premature Infant Pain Profile to assess pain during intramuscular injection of vitamin $K$, venipuncture for the newborn screening test and the first 3 heel lances for glucose monitoring (newborns of diabetic mothers only). Scores ranged from from 0 (no pain) to 18 (maximum pain).

Results: We included 240 newborns (120 from diabetic mothers, 120 from nondiabetic mothers). The overall mean pain score was lower among newborns who received sucrose than among those who received a placebo (mean difference $-1.3,95 \%$ confidence interval $[\mathrm{Cl}]-2.0$ to -0.6$)$. We found that pain scores during intramuscular injection did not differ significantly between the sucrose and placebo groups for newborns of diabetic or nondiabetic mothers (newborns of nondiabetic mothers: mean difference -1.1 , $95 \% \mathrm{Cl}-2.4$ to 0.2 ; newborns of diabetic mothers: mean difference $-1.0,95 \% \mathrm{Cl}-2.4$ to 0.4 ). During venipuncture, newborns who received sucrose had lower pain scores compared with those who received a placebo (newborns of nondiabetic mothers: mean difference $-3.2,95 \% \mathrm{Cl}-4.6$ to -1.8 ; newborns of diabetic mothers: mean difference -2.4 , $95 \% \mathrm{Cl}-3.8$ to -1.0$)$. Among newborns of diabetic mothers, there was no difference in pain during the first 3 heel lances or mean glucose levels between the sucrose and placebo groups ( $p=0.94$ and $p=0.29$ respectively).

Interpretation: We found a modest reduction of pain in newborns of both diabetic and nondiabetic mothers when sucrose was used for all medical procedures performed in the first 2 days after birth. However, when each procedure was analyzed separately, we found that the effectiveness of sucrose was limited to venipuncture for the newborn screening test. (Clinicaltrials.gov trial register no. NCT00213213.)

Une version française de ce résumé est disponible à l'adresse www.cmaj.ca/cgi/content/full/179/1/37/DC1

CMAJ 2008;179(1):37-43
A 11 newborns experience pain from medical procedures in the first days after birth. At the very least, newborns receive an intramuscular injection of vitamin $\mathrm{K}$ and venipuncture or heel-lance for newborn screening tests. Newborns of diabetic mothers, about $4 \%$ of newborns, undergo additional heel-lances for monitoring glucose levels. ${ }^{1,2}$ Untreated pain from these procedures can have long-lasting effects. ${ }^{3,4} \mathrm{We}$ previously reported that newborns of diabetic mothers who received an average of 10 heel-lances within 2 days of birth exhibited a heightened pain response during subsequent venipunctures compared with newborns from nondiabetic mothers. ${ }^{2}$

Studies aimed at reducing pain and its consequences in newborns are clinically important..$^{5-7}$ Sucrose (table sugar) has great potential for routine use. ${ }^{6}$ Sucrose is a naturally occurring sweetener with analgesic effects in newborns. Although not fully understood, the mechanism of action is thought to involve activation of the endogenous opioid system through taste. ${ }^{8}$ This is supported by the presence of opioid receptors on the tongue and animal studies showing that analgesia can be reversed by opioid antagonists during noxious stimulation. ${ }^{9}$ Moreover, chronic consumption of sweeteners has been shown to negate the analgesic effects of opioids. ${ }^{10}$

In systematic reviews, single doses of sucrose have been reported to reduce pain in newborns undergoing commonly performed medical procedures, including heel-lancing and venipuncture. ${ }^{11,12}$ Based on these data, national and international pain management guidelines promote the widespread use of sucrose. ${ }^{13-16}$ However, sucrose has not been evaluated for all commonly performed procedures in healthy term newborns, and there are concerns that sucrose may raise blood glucose levels in newborns of diabetic mothers. It is necessary to demonstrate that sucrose is effective and safe for varied procedures, repeated use and diverse infant populations.

We sought to evaluate the effectiveness and safety of sucrose in newborns of diabetic mothers and nondiabetic moth-

From the Leslie Dan Faculty of Pharmacy (Taddio, Smith), the Faculty of Medicine (Shah, Koren), the Faculty of Nursing (Stevens), the Departments of Anesthesia (Katz) and Population Health Sciences (Stephens, Beyene), and the Institute of Medical Sciences (Hancock), University of Toronto; Child Health Evaluative Sciences (Taddio, Hancock, Smith, Stephens, Atenafu, Beyene, Koren, Stevens), The Hospital for Sick Children; The Department of Paediatrics (Shah), Mount Sinai Hospital; and the Department of Psychology (Katz), York University, Toronto, Ont. 
ers undergoing routine painful medical procedures during the first 2 days after birth. Our hypothesis was that sucrose reduces pain during intramuscular injection of vitamin $\mathrm{K}$, venipuncture for the newborn screening test and heel lance for blood glucose monitoring without serious adverse effects.

\section{Methods}

\section{Study design and population}

We conducted a double-blind randomized controlled trial at Mount Sinai Hospital, Toronto, Ontario. We recruited participants between Sept. 15, 2003, and July 27, 2004. We included healthy newborns ( $\geq 36$ weeks gestation) born to nondiabetic mothers with uneventful pregnancies or to diabetic mothers (type 1, type 2, or gestational diabetes that was controlled by diet or insulin). We identified eligible newborns from the daily logs in the delivery suite. We excluded newborns with major congenital or neurologic anomalies, or clinical diagnoses of birth asphyxia ${ }^{17}$ or seizures. We also excluded newborns admitted to the neonatal intensive care unit, those who received analgesics or sedatives or both, and those who were scheduled to undergo circumcision during the study period.

This study was approved by the research ethics boards of Mount Sinai Hospital, The Hospital for Sick Children and York University, Toronto, Ontario, as well as Health Canada. A member of the study team obtained written consent to participate from the parents before the birth of the newborn.

\section{Intervention}

Newborns received $2 \mathrm{~mL}$ of a $24 \%$-sucrose solution ${ }^{11}$ or $2 \mathrm{~mL}$ of sterile water (placebo) by mouth before all intramuscular injections, venipunctures and heel-lances performed within 2 days of birth. Beginning 2 minutes before the procedure, the nurse assigned to each newborn used a syringe to administer sucrose or placebo to the anterior surface of the tongue over a period of about 60 seconds. All procedures were performed by certified nurses or physicians, as per standard practices at the study centre.

\section{Concealment of allocation}

Concealment of allocation was achieved by carrying out randomization and dispensing functions off-site. Using a random numbers table and allocation ratio of 1:1 for sucrose and placebo, a research pharmacist at The Hospital for Sick Children prepared 2 separate block-randomization assignments (1 for newborns of diabetic mothers and 1 for newborns of nondiabetic mothers). Respironics (Murrysville, Pennsylvania) provided single-use sucrose (Sweet-Ease) and placebo packages that appeared identical. The solutions were labelled with each newborn's name and stored in patient-specific plastic bags in the newborn's medication bin. The study personnel, health care workers and parents did not know which newborns received sucrose. The success of blinding was not evaluated.

\section{Pain outcomes}

The primary outcome measure was pain. We videotaped all newborns during intramuscular injection of vitamin $\mathrm{K}$ performed in the first hour after birth and during a venipuncture performed after at least 24 hours for the newborn screening test. As well, we videotaped newborns of diabetic mothers during the first 3 heel-lances that were performed within the first 12 hours after birth as part of the glucose monitoring protocol at the study centre.

About 5 minutes before each procedure, a portable pulse oximeter was applied to the newborn's foot or hand, and a digital video camera was mounted on a tripod 1 metre from the newborn's body. Throughout the procedure, we videotaped the newborn's face and recorded his or her physiologic responses (heart rate, oxygen saturation). All newborns were in a supine position and were partially swaddled (the limb that was used for the procedure was exposed). Parents were present during the procedure but did not touch or interact with their newborn.

We assessed pain using a validated composite pain measure (Premature Infant Pain Profile). ${ }^{18}$ This profile includes 3 facial actions (brow bulge, eyes squeezed shut, nasolabial furrow), and 2 physiologic (heart rate, oxygen saturation) and 2 contextual (gestational age, infant state) indices of pain. Using the videotaped images, a research assistant who was unaware of treatment allocation, study hypotheses and whether the newborn was from a diabetic or nondiabetic mother scored each facial action as present or absent in 2-second intervals for the first 30 seconds of the venipuncture. For intramuscular injection and heel lances, which were of shorter duration, only facial actions during the first 20 seconds were scored (or less if the procedure lasted less than 20 seconds). The percentage of the total time that each facial action was observed during each phase was calculated. For physiologic data, changes in heart rate and oxygen saturation from baseline were recorded over the same period. Using established methods, ${ }^{18}$ we calculated total Premature Infant Pain Profile scores for each procedure by summing the scores of the 7 indicators. Scores ranged from 0 (no pain) to 18 (maximum pain). We assessed inter-rater reliability by having a second rater score $28 \%$ of the procedures; the intraclass correlation coefficient was 0.97 (95\% confidence interval [CI] 0.96 to 0.98 ).

\section{Safety outcomes}

The adverse events monitored over the course of the study included spitting up within 30 minutes of medication being administered and blood glucose levels (among newborns of diabetic mothers only). A safety committee was convened to oversee serious adverse events (aspiration, prolonged desaturation).

\section{Sample size calculation and statistical analysis}

We based the sample size calculation on a clinically important difference of $20 \%$ in pain scores between the sucrose and placebo groups. ${ }^{19}$ Using previous data, ${ }^{2,20}$ we translated this to a moderate effect size (0.6) and a sample size of 45 newborns per group (total sample size $=90$ ). ${ }^{21}$ We doubled the sample size to 180 newborns because we planned to include 2 groups of newborns (those from diabetic and nondiabetic mothers) and increased the sample size to 240 to account for potential missing data and drop-outs. Thus, we included 60 newborns in each study group (newborns of diabetic mothers given sucrose, newborns of diabetic mothers given placebo, newborns of nondiabetic mothers given sucrose, newborns of nondiabetic mothers given placebo). 
In our primary analysis, we compared the average of all pain scores for each newborn in the sucrose and placebo groups using the Student $t$ test. Using this test, we separately compared pain scores between those who received sucrose and those who received a placebo for each group (newborns of diabetic or nondiabetic mothers) and for each procedure (intramuscular injection, venipuncture). We analyzed pain scores during the first 3 heel lances in newborns of diabetic mothers using a repeated- measures linear mixed-model analysis of variance.

We performed post-hoc analyses after adjusting for baseline characteristics (sex, mode of delivery, drugs given during delivery, gestational age, postnatal age) by use of a general linear model (for intramuscular injection and venipuncture) and a linear mixed-model analysis (for heel-lances). For newborns of diabetic mothers, we also included indices of maternal illness (type of diabetes, use of insulin, glycosylated he-

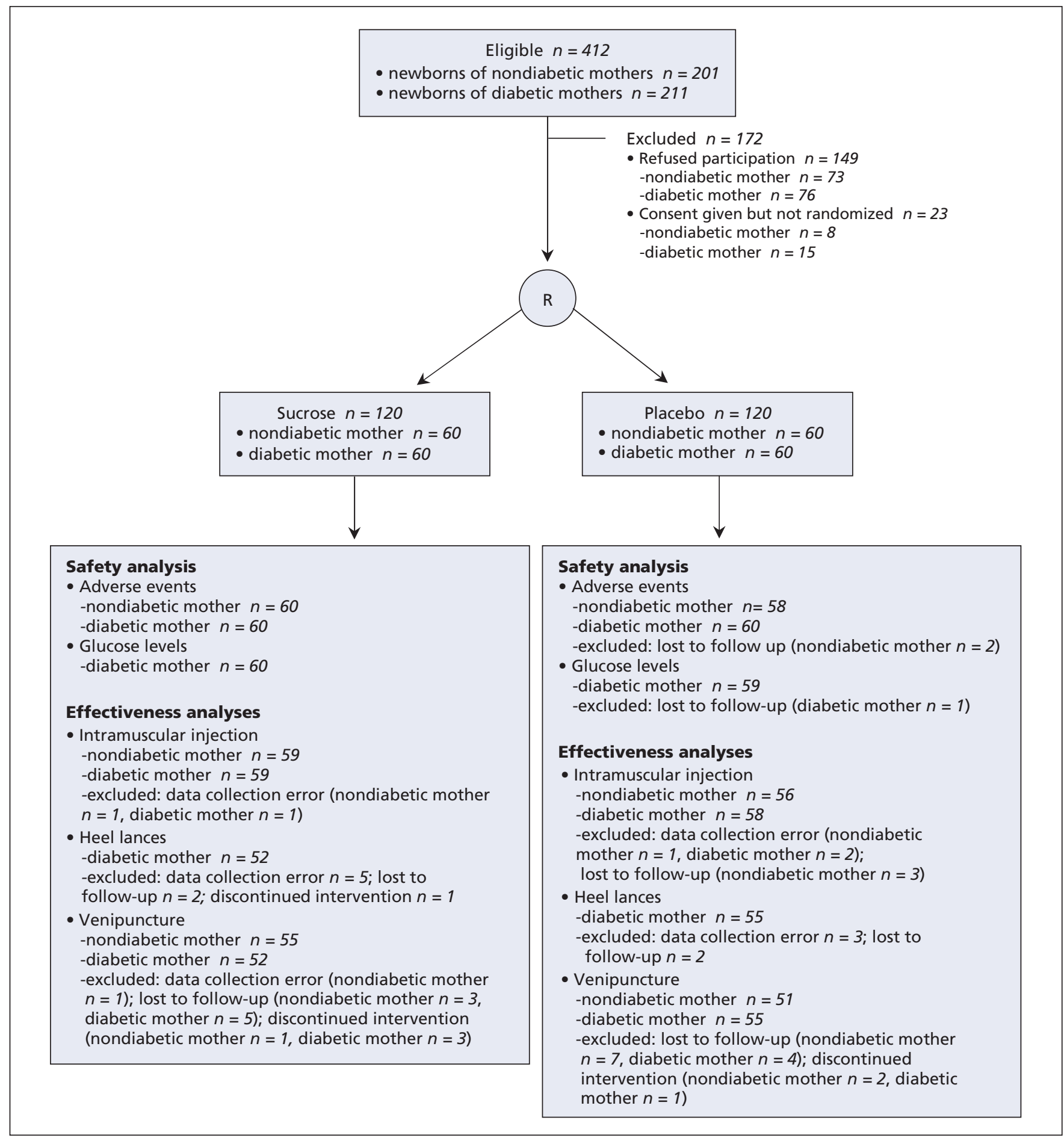

Figure 1: Flow of participants through the study. Note: $\mathrm{R}=$ randomization. 
moglobin concentration) and newborn blood glucose level at the time of the procedure (for heel-lances). We analyzed adverse events using the $\chi^{2}$ test or the Student $t$ test. An intentto-treat approach was used that included all infants with data. A $p$ value of 0.05 or less was considered significant.

\section{Funding and commercial interests}

The sucrose (Sweet-Ease) and placebo solutions used in this study were provided by Respironics Inc. The company provided no other support, nor did it influence the design, conduct or reporting of the trial.

\section{Results}

\section{Recruitment and withdrawals}

In total, we approached 412 parents, 263 of whom agreed to participate. Twenty-three newborns were not randomized;
6 had an unstable condition at birth and were withdrawn, and 17 were missed by study personnel. In total, 240 newborns were included in our study (120 in the sucrose group and 120 in the placebo group) (Figure 1).

\section{Birth characteristics and treatment-related factors}

Birth characteristics are shown in Table 1. Newborns of diabetic mothers received more heel lances before venipuncture and therefore received more doses of study medication (mean 6.4, standard deviation [SD] 2.0) than newborns of nondiabetic mothers (mean 2.3, SD 0.8) $(p<0.001)$.

\section{Pain response}

Our primary analysis showed that the overall mean score for the Premature Infant Pain Profile was significantly lower among newborns who received sucrose (mean score 6.8, SD 2.9) than among those who received a placebo (mean score 8.1, SD 2.5)

Table 1: Characteristics of newborns included in the randomized controlled trial of sucrose as an analgesic during painful procedures

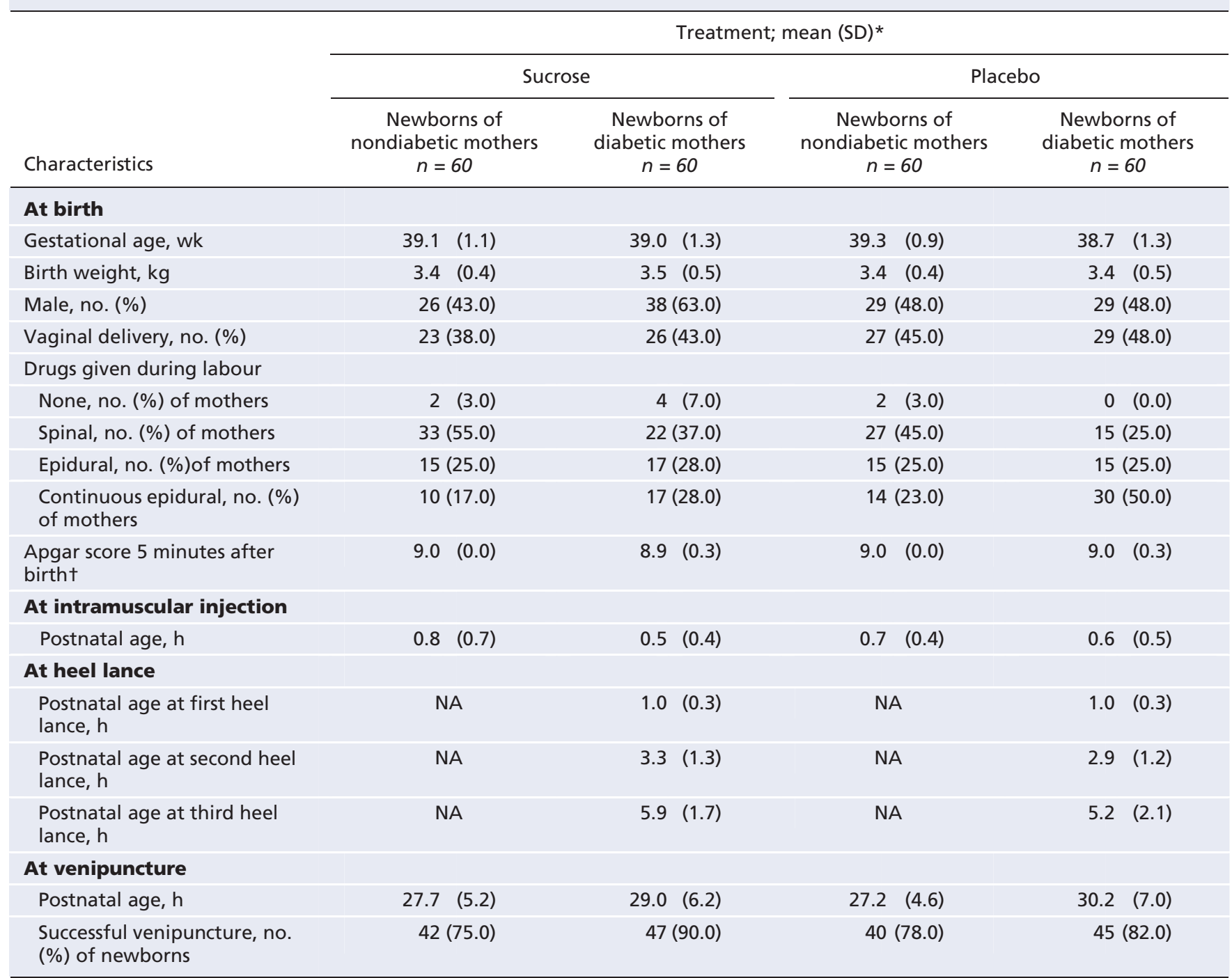

NA = not applicable.

*Unless stated otherwise.

tThe Apgar score was used to assess newborn health. Scores are based on 5 criteria (appearance, pulse, grimace, activity, respiration) and range from 0 to 10 . A score over 7 is generally considered to be healthy. 
(mean difference $-1.3,95 \% \mathrm{CI}-2.0$ to $-0.6, p<0.001$ ).

Pain scores during intramuscular injection, venipuncture and heel-lance (newborns of diabetic mothers only) are shown in Table 2. Pain scores during intramuscular injection did not differ between the sucrose and placebo groups for newborns of nondiabetic mothers (mean score $7.4,95 \% \mathrm{Cl}$ 6.4-8.4 v. mean score $8.5,95 \%$ CI 7.6-9.3, $p=0.10$ ) or diabetic mothers (mean score $6.2,95 \%$ CI $5.2-7.2$ v. mean score $7.2,95 \%$ CI $6.3-8.2, p=0.15$ ). During venipuncture, pain scores were significantly lower among newborns of nondiabetic mothers who received sucrose compared with those who received a placebo (mean score $5.7,95 \%$ CI 4.7-6.7 v. mean score $8.9,95 \%$ CI 7.9-9.9, $p<0.001$ ). This was consistent among newborns of diabetic mothers (sucrose group: mean score 6.8, 95\% CI 5.7-7.9 v. placebo: mean score 9.2, 95\% CI 8.4-10.1, $p<0.001)$. Among newborns of diabetic mothers, the pain scores during the first 3 heel lances did not differ between the sucrose and placebo groups.

Post-hoc analyses that adjusted for baseline characteristics did not change the pattern of the results.

\section{Adverse effects}

Among newborns of nondiabetic mothers, more newborns in the placebo group spat up $(p=0.04)$. Among newborns of diabetic mothers, there was no difference in the incidence of minor adverse effects or mean blood glucose levels between those who received sucrose or placebo (Table 3 ). There were no serious adverse events during the study.

\section{Interpretation}

We found that sucrose reduced overall pain in newborns when administered before painful medical procedures during the first 2 days after birth. The observed difference in pain, however, was modest $(16 \%)$ and did not meet our a priori definition of a clinically important difference of $20 \% .{ }^{19}$ When we analyzed each procedure separately for newborns of diabetic and nondiabetic mothers, the results were consistent between the 2 groups of newborns but the results were inconsistent between procedures. In both newborn groups, sucrose was effective for reducing pain during venipuncture for the newborn screening test, but it was ineffective during intramuscular injection of vitamin K. In addition, sucrose was ineffective for reducing pain in newborns of diabetic mothers during repeated heel-lances.

Our observation that sucrose reduces pain during venipuncture is consistent with previous research involving term newborns. ${ }^{22}$ Unexpectedly, we did not observe analgesic effects during either intramuscular injection of vitamin $\mathrm{K}$ in either group or during repeated heel-lancing for blood glucose monitoring in newborns of diabetic mothers. In contrast, previous studies reported analgesic effects in newborns undergoing heel lancing ${ }^{12}$ and in older infants undergoing intramuscular injections of vaccines. ${ }^{23-25}$ To rule out an effect of baseline newborn and maternal characteristics on the effectiveness of sucrose, we performed post-hoc analyses adjusted for birth characteristics. Among newborn of diabetic mothers, we also adjusted for indices of illness. We found no significant effects of any of these factors on the effectiveness of sucrose, which strengthens our interpretation of the results.

The observed ineffectiveness of sucrose during intramuscular injection and heel lances may be because of newborn postnatal age, method of pain assessment, and procedure technique. In our study, all procedures except for venipuncture were performed within 12 hours of birth. In contrast, previous studies have reported the effects of sucrose beyond 1 day of birth. ${ }^{12}$ Significant concentrations of endogenous opioids ( $\beta$-endorphin) are present in newborns during birth adaptation in the first hours after birth. ${ }^{26,27}$ It is possible that sucrose did not offer any additional benefit above physiologic

Table 2: Scores obtained on the Premature Infant Pain Profile during intramuscular injection, venipuncture and heel lance for newborns included in the randomized controlled trial of sucrose as an analgesic during painful procedures

Treatment group; mean pain score* $(95 \% \mathrm{Cl})$

\begin{tabular}{|c|c|c|c|c|}
\hline \multirow[b]{2}{*}{ Group } & \multirow{2}{*}{ Sucrose } & & \multirow[b]{2}{*}{ Mean difference $(95 \% \mathrm{Cl})$} & \multirow[b]{2}{*}{$p$ value } \\
\hline & & Placebo & & \\
\hline \multicolumn{5}{|c|}{$\begin{array}{l}\text { Newborns of nondiabetic } \\
\text { mothers }\end{array}$} \\
\hline Venipuncturet & 5.7 (4.7 to 6.7$)$ & 8.9 (7.9 to 9.9$)$ & $-3.2(-4.6$ to -1.8$)$ & $<0.001$ \\
\hline \multicolumn{5}{|c|}{ Newborns of diabetic mothers } \\
\hline Second heel lance $¥$ & 8.2 (7.0 to 9.3$)$ & $6.9(5.8$ to 7.9$)$ & - & \\
\hline Third heel lance $\ddagger$ & $6.9(5.9$ to 8.0$)$ & 7.5 (6.5 to 8.6$)$ & - & \\
\hline Venipuncturet & $6.8(5.7$ to 7.9$)$ & $9.2(8.4$ to 10.1$)$ & $-2.4(-3.8$ to -1.0$)$ & $<0.001$ \\
\hline
\end{tabular}

Note: $\mathrm{Cl}=$ confidence interval.

*Pain was measured using the Premature Infant Pain Profile, which includes 7 indicators of pain (brow bulge, eyes squeezed shut, nasolabial furrow, gestational age, infant state, and changes in heart rate and oxygen saturation from baseline).

†Pain scores during intramuscular injection and venipuncture were compared between groups using the Student $t$ test.

¥Pain scores during heel lances were compared using mixed-model analysis.

§Mean difference $(95 \% \mathrm{Cl})$ refers to overall value. 
Table 3: Adverse events observed among newborns included in the randomized controlled trial of sucrose as an analgesic during painful procedures

\begin{tabular}{|c|c|c|c|}
\hline \multirow[b]{2}{*}{ Adverse event } & \multicolumn{2}{|c|}{ Treatment; no. (\%)* of newborns } & \multirow[b]{2}{*}{$p$ valuet } \\
\hline & Sucrose & Placebo & \\
\hline \multicolumn{4}{|l|}{$\begin{array}{l}\text { Newborns of nondiabetic } \\
\text { mothers }\end{array}$} \\
\hline Spitting up & $2(3)$ & $8(14)$ & 0.04 \\
\hline \multicolumn{4}{|c|}{ Newborns of diabetic mothers } \\
\hline Spitting up & $7(12)$ & $9(15)$ & 0.59 \\
\hline $\begin{array}{l}\text { Blood glucose level, } \mathrm{mmol} / \mathrm{L} \text {, } \\
\text { mean (SD) }\end{array}$ & $3.4(0.6)$ & $3.2(0.7)$ & 0.29 \\
\hline
\end{tabular}

levels of $\beta$-endorphin. The analgesic effect of sucrose may also depend on when pain is assessed. In the present study, we measured pain during the procedure, whereas other studies assessed pain for up to several minutes afterward. ${ }^{12} \mathrm{Su}-$ crose may be a relatively weak analgesic ${ }^{28}$ that is more effective at calming infants during the recovery period after a painful procedure than during the actual procedure when pain is at its peak. ${ }^{29}$ Finally, we used a fully retractable automatic lancet during the heel-lances and collected only a single drop of blood. This resulted in minimal pain in newborns ${ }_{1}^{18}$ which was perhaps too low for sucrose to have a measurable effect.

There is debate about the use of placebos and the ethics of withholding treatment in clinical trials evaluating analgesics. ${ }^{30}$ It is important, however, that the benefits of an analgesic be established before it becomes the standard of care. Sucrose is quickly becoming the standard of care in nurserie ${ }^{31}$ because of the ease of administration, affordability and perceived safety and efficacy. It is even replacing established analgesics such as opioids and local anesthetics. ${ }^{32}$ We used a placebocontrolled design in our study because of unanswered questions about the effectiveness of sucrose for different procedures and when used repeatedly, and because the official standard of care at our institution did not include sucrose or any other analgesic intervention. Although our results show that sucrose is relatively safe, its effectiveness in the first 2 days after birth is limited and cannot be extrapolated from one painful procedure to another.

The present study has several strengths, including its large sample size and double-blind randomized design. Even for a medium effect size of 0.4 , we had greater than $95 \%$ power to detect a difference using a repeated-measures design with $\alpha=0.05$. Moreover, our results are generalizable to most clinical settings because we included a diverse infant population and the most commonly performed procedures, and because we used a "real world" approach that included multiple nurses and physicians administering study medications and performing procedures.

However, a limitation of our study is that we may have increased variability in pain responses by unsettling the newborns with the application of physiologic monitors. In addi- tion, our pain assessment tool, the Premature Infant Pain Profile, may not have adequately captured infant pain. However, that it is the best validated measure of pain in newborns. ${ }^{7}$ Our results are limited to the effectiveness of sucrose when administered as the sole analgesic agent. Pain relief is enhanced when sucrose is combined with a pacifier, ${ }^{16}$ but our hospital disallowed the use of pacifiers for the present study because of baby-friendly initiatives designed to encourage breast-feeding. Other institutions have similar practices. ${ }^{31}$

In conclusion, we found a modest $16 \%$ reduction in overall pain among newborns of both diabetic and nondiabetic mothers when sucrose was used repeatedly for all procedures performed in the first 2 days after birth. Moreover, when we evaluated each procedure separately, the effectiveness of sucrose was limited to venipuncture. Based on these results, we recommend using sucrose to reduce pain in newborns undergoing venipuncture for the newborn screening test but not for intramuscular injection of vitamin $\mathrm{K}$. In addition, sucrose is not recommended for heel lances for glucose monitoring in newborns of diabetic mothers. Current pain management guidelines should be updated to reflect the results of the present study. Further studies are needed to elucidate the mechanisms responsible for the ineffectiveness of sucrose immediately after birth.

This article has been peer reviewed.

Competing interests: None declared.

Contributors: All of the authors participated in the conception and design of the study, and the acquisition, analysis and interpretation of the data. All of the authors contributed to the drafting of the article or its critical revision for important intellectual content and gave final approval of the version to be published. The primary, corresponding author assumes responsibility for the entire manuscript. The corresponding author had full access to all data in the study, takes responsibility for the statistical analysis and for the decision to submit for publication.

Acknowledgements: We thank the staff at Mount Sinai Hospital and the parents of participating newborns. We thank Dr. Sunit Godambe, Ms. Jessica Singh and Ms. Amanda Levine for their help with data collection and data entry, Ms. Lucia Taddio and Mr. Erwin Darra for data coding, and Mr. A.L. Ilersich for critical review of this manuscript.

The sucrose (Sweet-Ease) and placebo solutions used in this study were provided by Respironics Inc (Murrysville, Pennsylvania). 
This study was supported by a Canadian Institutes of Health Research (CIHR) grant to Anna Taddio (MCT 63143) and by a Sick Kids Foundation graduate student Research Training Competition Award to Ryan Smith. Anna Taddio is supported by a New Investigator Award from the Canadian Institutes of Health Research, and Joel Katz is supported by a Canada Research Chair in Health Psychology at York University. The funding agencies did not provide any input regarding the design and conduct of the study; collection, management, analysis and interpretation of the data; or the preparation, review or approval of the manuscript.

\section{REFERENCES}

1. Williams AF. Hypoglycaemia of the newborn: a review. Bull World Health Organ 1997; 75:261-90.

2. Taddio A, Shah V, Gilbert-MacLeod C, et al. Conditioning and hyperalgesia in newborns exposed to repeated heel lances. JAMA 2002;288:857-61.

3. Taddio A, Katz J. The effects of early pain experience in full-term and preterm neonates on subsequent pain responses later in infancy and childhood. Paediatr Drugs 2005;7:245-57.

4. Grunau RE. Long-term consequences of pain in human neonates. In: Anand KJS, Stevens BJ, McGrath PJ, editors. Pain in neonates: pain research and clinical management. 2nd ed. (vol. 10). London; Elsevier: 2000. p. 55-76.

5. World Health Organization. Promoting safety of medicines for children. Geneva: The Organization; 2007. Available: www.who.int/medicines/publications/essential medicines/Promotion_safe_med_childrens.pdf (accessed 2008 Apr 9).

6. Anand KJS, Johnston CC, Oberlander T, et al. Analgesia and local anesthesia during invasive procedures in the neonate. Clin Ther 2005;27:844-76.

7. Anand KJS, Aranda JV, Berde CB, et al. Summary proceedings from the neonatal pain-control group. Pediatrics 2006;117:S9-22.

8. Blass EM, Watt LB. Suckling- and sucrose-induced analgesia in human newborns. Pain 1999;83:611-23

9. Shide DJ, Blass EM. Opioid-like effects of intraoral infusions of corn oil and polycose on stress reactions in 10-day-old rats. Behav Neurosci 1989;103:1168-75.

10. Lieblich I, Cohen E, Ganchrow J. Morphine tolerance in genetically selected rats induced by chronically elevated saccharine intake. Science 1983;221:871-3.

11. Stevens B, Taddio A, Ohlsson A, et al. The efficacy of sucrose for relieving procedural pain in neonates - a systematic review and meta-analysis. Acta Paediatr 1997;86:837-42.

12. Stevens B, Yamada J, Ohlsson A. Sucrose for analgesia in newborn infants undergoing painful procedures. Cochrane Database Syst Rev 2004;(3):CD001069.

13. Committee on Fetus and Newborn, Committee on Drugs, Section on Anesthesiology, Section on Surgery, et al. Prevention and management of pain and stress in the neonate. Pediatrics 2000;105:454-61.

14. American Academy of Pediatrics and the Canadian Paediatric Society. Prevention and management of pain and stress in the neonate. Paediatr Child Health 2000;5:31-8 Available: http://aappolicy.aappublications.org/cgi/reprint/pediatrics;105/2/454.pdf (available 2008 May 30).

15. International Evidence-Based Group for Neonatal Pain. Consensus statement for the prevention and management of pain in the newborn infant. Arch Pediatr Adolesc Med 2001;155:173-80.
16. American Academy of Pediatrics, Canadian Paediatric Society. Prevention and management of pain in the neonate: an update. Pediatrics 2006;118:2231-41

17. American Academy of Paediatrics, American College of Obstetricians and Gynecologists. Relationships between perinatal factors and neurologic outcome. In: Poland RL, Freeman RK, editors. Guidelines for perinatal care. 3rd ed. Elk Grove Village (Il): American Academy of Paediatrics; 1992. p. 221-4.

18. Stevens B, Johnston C, Petryshen P, et al. Premature infant pain profile: development and initial validation. Clin J Pain 1996;12:13-22.

19. Shah V, Ipp M, Sam J, et al. Eliciting the minimal clinically important difference in the pain response from parents of newborn infants and nurses [abstract]. Annual Pediatric Academic Societies meeting; 2004 May 1-4; San Francisco (CA). Pediatr Res 2004;55:519A.

20. Stevens B, Yamada J, Beyene J, et al. Consistent management of repeated procedural pain with sucrose in preterm neonates: Is it effective and safe for repeated use over time? Clin J Pain 2005;21:543-8.

21. Cohen J. Statistical power analysis for the behavioral sciences. 2nd ed. Hillsdale (NJ): Lawrence Erlbaum Associates; 1988.

22. Carbajal R, Chauvet X, Couderc S, et al. Randomised trial of analgesic effects of sucrose, glucose, and pacifiers in term neonates. BMJ 1999;319:1393-7.

23. Allen KD, White DD, Walburn JN. Sucrose as an analgesic agent for infants during immunization injections. Arch Pediatr Adolesc Med 1996;150:270-4.

24. Ramenghi LA, Webb AV, Shevlin PM, et al. Intra-oral administration of sweettasting substances and infants' crying response to immunization: a randomized, placebo-controlled trial. Biol Neonate 2002;81:163-9.

25. Lewindon PJ, Harkness L, Lewindon N. Randomised controlled trial of sucrose by mouth for the relief of infant crying after immunization. Arch Dis Child 1998;78: 453-6.

26. Facchinetti F, Bagnoli F, Sardelli S, et al. Plasma opioids in the newborn in relation to the mode of delivery. Gynecol Obstet Invest 1986;21:6-11.

27. Facchinetti F, Bagnoli F, Bracci R, et al. Plasma opioids in the first hours of life. Pediatr Res 1982;16:95-8.

28. Brady-Fryer B, Wiebe N, Lander JA. Pain relief for neonatal circumcision. Cochrane Database Syst Rev 2004;(4):CD004217.

29. Barr RG, Young SN, Wright JH, et al. "Sucrose analgesia" and diphtheria-tetanuspertussis immunizations at 2 and 4 months. J Dev Behav Pediatr 1995;16:220-5.

30. Anand KJ, Aranda JV, Berde CB, et al. Analgesia and anesthesia for neonates: study design and ethical issues. Clin Ther 2005;27:814-43.

31. Taddio A, Yiu A, Smith RW, et al. Utilization of sweetening agents for procedural pain management in neonates in Canada [poster presentation]. Annual Pediatric Academic Societies meeting; 2008 May 2-6; Honolulu, Hawaii. Available: www .abstracts2view.com/pas/view.php?nu=PAS08L1_265 (accessed 2008 June 2).

32. Taddio A, Crosdale B, Hogan ME, et al. Safety of morphine in non-intubated infants in the neonatal intensive care unit [poster presentation]. Annual Pediatric Academic Societies meeting; 2008 May 2-6; Honolulu, Hawaii. Available: www.abstracts2view .com/pas/view.php?nu=PAS08L1_488 (accessed 2008 June 2).

Correspondence to: Dr. Anna Taddio, Associate Professor, Leslie Dan Faculty of Pharmacy, University of Toronto, 144 College St., Toronto ON M5S 3M2; fax 416 978-1833;

anna.taddio@utoronto.ca

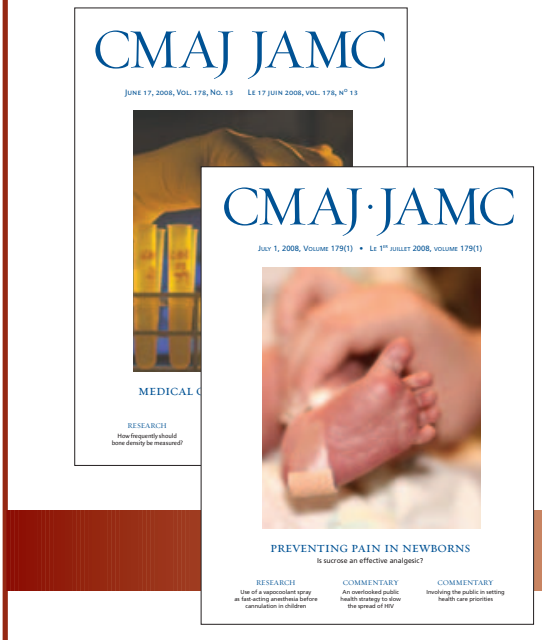

\section{Online manuscript submission and peer review available for $C M A J$}

